\title{
Herstellung endlosfaserverstärkter, thermoplastischer Halbzeuge für Karosseriestrukturbauteile in Großserie
}

Daniel Hofbauer ${ }^{1)}$, Norbert Schramm²), Lothar Kroll3), Joerg Kaufmann ${ }^{4)}$

1) Konzeptplanung CFK, BMW Group, 84030 Landshut, Deutschland

2) Professur Strukturleichtbau und Kunststoffverarbeitung, Technische Universität Chemnitz, Reichenhainer Straße 31/33, 09126 Chemnitz, Deutschland

3) Professur für Mechanik und Maschinenkonstruktion, Technische Universität Opole, ul. S. Mikołajczyka 5, 45271 Opole, Polen

4) Professur Textile Technologien, Technische Universität Chemnitz, Reichenhainer Straße 31/33, 09126 Chemnitz, Deutschland

\section{Stichworte}

Endlosfaserverstärkte thermoplastische Halbzeuge, Karosseriestrukturbauteil, Konsolidierung, Doppelbandpresse, Schwellerprofil

\begin{abstract}
Derzeit werden in der Automobilindustrie Faser-Kunststoff-Verbunde mit thermoplastischer Matrix nur für semistrukturelle Bauteile mit geringeren Steifigkeits- und Festigkeitsanforderungen als Strukturbauteile und ohne Einbindung in die Hauptlastpfade einer Karosseriestruktur eingesetzt. Auf Basis von hohen mechanischen Anforderungen an ein Schwellerprofil einer Karosserie soll demgegenüber eine zweischalige Bauweise unter Verwendung von mit Polyamid-Matrix vorimprägnierten, unidirektionalen Kohlenstofffasertapes zur Anwendung kommen. Infolge der extremen Belastungen ergibt sich ein Laminataufbau mit einer hohen Vorzugsrichtung in $0^{\circ}$-Faserorientierung und einer Dicke von bis zu $4,9 \mathrm{~mm}$. Zur Herstellung derart hoher Wandstärken müssen die einzelnen unidirektionalen Tapes schichtweise aufgebaut und zu einem Laminat konsolidiert werden. Die Konsolidierung der Einzellagen kann dabei entweder direkt bei deren Ablage, auf statischen Heizpressen oder mittels Doppelbandpressen erfolgen. Für eine großserielle Herstellung ist aufgrund des kontinuierlichen Fertigungsprinzips eine Doppelbandpresse grundsätzlich von Vorteil. Anhand von experimentellen Fertigungsversuchen zur Konsolidierung der Layups konnte jedoch weder mit einer isobaren Doppelbandpresse, noch mit einer statischen Laborplattenpresse eine ausreichend gute Laminatqualität erzeugt werden. Lediglich die Veränderung des Lagenaufbaus, verbunden mit einer Reduzierung der mechanischen Eigenschaften des Laminats, erzeugte auf der Laborplattenpresse eine entsprechende Qualität. Allerdings ist die dafür erforderliche Zykluszeit für eine Großserienfertigung inakzeptabel. Somit stellt weder der untersuchte Doppelbandprozess, noch der statische Pressprozess hinsichtlich der erzielbaren Laminatqualität und der erforderlichen Anlagenkapazität für das beschriebene Schwellerprofil eine großserientaugliche Fertigungstechnologie dar. Darauf aufbauend müssen weiterführende Fertigungsstudien zum Doppelbandpressprozess mit Anpassung der Prozessparameter durchgeführt werden.
\end{abstract}




\section{Automobile Anwendungen in strukturellen und semi-strukturellen Komponenten}

Endlosfaserverstärkte Kunststoffe leisten aufgrund ihrer hervorragenden gewichtsspezifischen Eigenschaften einen wichtigen Beitrag zur Reduzierung des Gesamtfahrzeuggewichtes. Nicht zuletzt durch den Einsatz von kohlenstofffaserverstärkten Kunststoffen (CFK) kann beispielsweise das Gewicht der Karosseriestruktur des BMW 7er um $130 \mathrm{~kg}$ im Vergleich zum Vorgänger verringert werden [1, 2]. Wie auch bei den Strukturbauteilen des BMW i3, BMW i8 und Audi A8 kommen bisher jedoch nur FaserKunststoff-Verbunde (FKV) mit duromeren Matrixsystemen zum Einsatz. Hingegen werden FKV mit thermoplastischer Matrix (TP-FKV) aktuell nur für semistrukturelle Bauteile mit geringeren Steifigkeitsund Festigkeitsanforderungen und ohne strukturfeste Einbindung in die Hauptlastpfade einer Karosseriestruktur, wie zum Beispiel dem Stoßfängerträger des BMW M3 [3], dem Frontendträger des Audi A8 [3], dem Batterieträger des Audi R8 e-tron [4], der Sitzschale des Opel Astra OPC [5] und einer Durchlade, welche in Zusammenarbeit der Brose Gruppe und der TU Chemnitz entwickelt wurde [6,7], eingesetzt. Dabei werden sowohl endlos-, lang- als auch kurzfaserverstärkte Kunststoffe kombiniert miteinander verarbeitet und somit beispielsweise beim Stoßfängerträger des BMW M3 eine Gewichtsreduzierung von rund $60 \%$ im Vergleich zum Referenzbauteil erzielt [3]. Die Durchlade erreicht ebenfalls eine Gewichtsreduzierung um circa $60 \%$ und eine Reduzierung der Herstellungskosten von etwa $30 \%$ gegenüber dem metallischen Referenzbauteil [6]. Für diese semistrukturellen Anwendungen werden zudem, anders als bei den beschriebenen Strukturbauteilen, überwiegend FKV mit Glasfasern in Form von vorimprägnierten, faserverstärkten Halbzeugen verwendet.

Im Folgenden soll das Potenzial von thermoplastischen FKV auch für strukturelle Anwendungen, auf Basis der funktionalen Anforderungen an ein Schwellerprofil, untersucht werden. Der Schweller ist ein fester Bestandteil der Karosseriestruktur, der auf beiden Fahrzeugseiten jeweils unterhalb des Türeinstiegs angeordnet ist. Er trägt maßgeblich zur Steifigkeit der Karosserie bei und schützt die Insassen bei einem seitlichen Pfahlcrash [8, 9, 10]. Aufgrund der hohen Belastungen, die von derartigen Strukturbauteilen zu übertragen sind, werden sehr hohe mechanische Eigenschaften gefordert. Zum Einsatz kommen daher ausschließlich CFK aus unidirektionalen, vorimprägnierten Tapes (UD-Tapes) und Langfaser-Spritzgussgranulat. Als Matrixwerkstoff der TP-FKV wird jeweils ein Polyamid 66 (PA66) verwendet (siehe Tab. 1).

Tabelle 1: Faserarten, -längen, -massenanteile und Matrixwerkstoff des TP-FKV-Schwellerprofils

\begin{tabular}{cccccc}
\hline Nr. & Faserart & Faserlänge & Halbzeug & Fasermassenanteil [\%] & Matrix \\
\hline 1 & Kohlenstofffaser & Endlos & UD-Tape & 60 & PA66 \\
2 & Kohlenstofffaser & Lang & Stäbchengranulat & 40 & PA66 \\
\hline
\end{tabular}

\section{Anforderungen an das Strukturbauteil}

Die funktionalen Anforderungen an ein Schwellerprofil werden überwiegend durch den Crashlastfall eines seitlichen Pfahlaufpralls nach FMVSS 214 definiert und können, vereinfacht durch eine DreiPunkt-Biegebelastung des Profils, experimentell geprüft werden (siehe $A b b$. 1). Neben dieser Hauptbelastung trägt der Schweller auch zur globalen Karosseriesteifigkeit, wie beispielsweise der Torsionssteifigkeit, bei, welche aber für die Dimensionierung des Schwellers eine untergeordnete Rolle spielt. Aufgrund dessen dient die beschriebene Biegebelastung als Grundlage für die Gestaltung und virtuelle Auslegung des Profils [11, 12]. In Anlehnung an das Crashkonzept der Fahrzeuge BMW i3 und BMW i8 wird zur Energieabsorption zusätzlich eine deformierbare, thermoplastische Wabenstruktur in der Bauteilauslegung mit betrachtet, welche die Kraft des Impaktors flächig auf das thermoplastische TP-FKV-Profil verteilt (siehe auch [10]). 

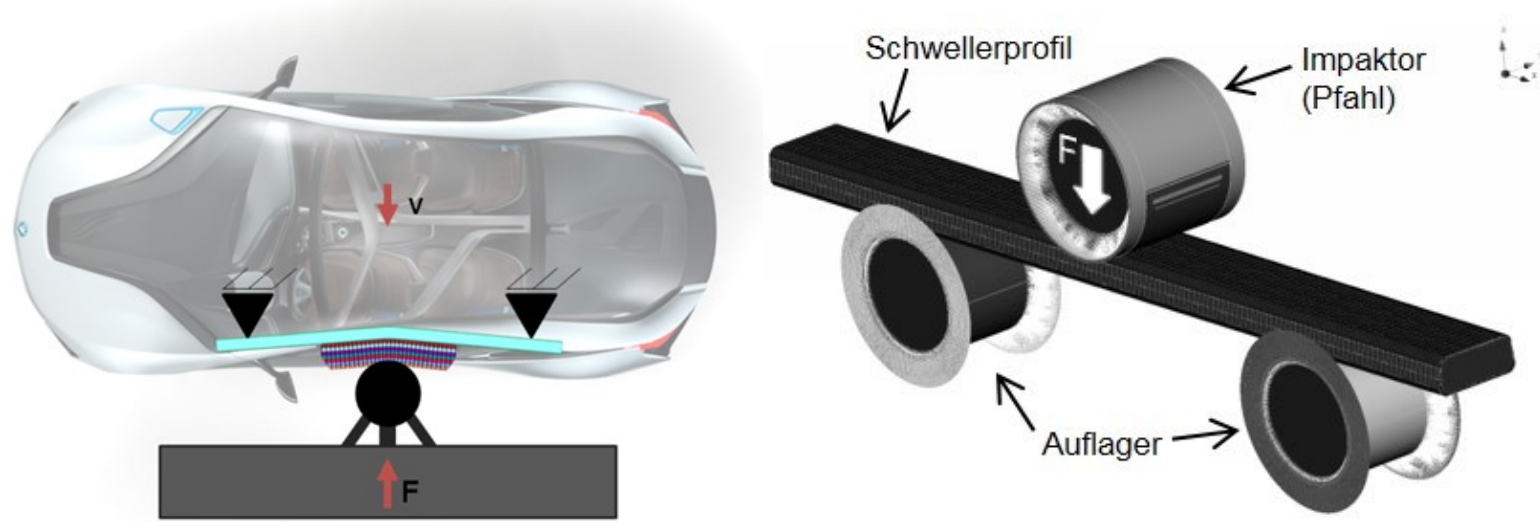

Abbildung 1: Belastungsart des Fahrzeugschwellers bei seitlichem Pfahlaufprall und abgeleiteter Ersatzlastfall anhand des BMW i8 [11, 12]

Als funktionale Referenzgeometrie für die virtuelle Auslegung des TP-FKV-Konzeptes dient ein Schwellerprofil, welches ein triaxiales Geflecht als textile Verstärkungsstruktur besitzt. Dabei werden die axial ausgerichteten Fasern aus 50K Kohlenstofffasern und die Flechtfäden im Faserwinkel von $+/-45^{\circ}$ zur Bauteillängsachse aus Glasfasern gebildet (siehe [2, 11]). Der Umflechtkern besteht aus einem Polyurethanschaum mit einer Dichte von $200 \mathrm{~g} / \mathrm{l}$, der zur funktionalen Verstärkung nach dem Herstellungsprozess im Bauteil verbleibt. Im Anschluss an den Flechtprozess wird das textile Halbzeug im Resin-Transfer-Moulding-Verfahren (RTM) mit einem Epoxidharz-/Härter-Gemisch getränkt und die Matrix anschließend ausgehärtet.

Anhand einer analytischen Steifigkeitsbetrachtung wurde zuerst das ideelle Flächenträgheitsmoment des TP-FKV-Konzeptes, unter Beachtung der Materialwertigkeiten, einem Verhältnis der E-Moduln der verwendeten Werkstoffe, dem Referenzbauteil gegenübergestellt (siehe [12]). Anschließend erfolgte eine numerische Auslegung mit linear-elastischem Werkstoffverhalten, in Anlehnung an die maximale Kraft $F_{\max }$ von $54 \mathrm{kN}$ bei einem Deformationsweg von $28 \mathrm{~mm}$ im Vergleich zum Referenzbauteil.

\section{Bauteilkonzept und virtuelle Auslegung}

TP-FKV ermöglichen bei deren Verarbeitung eine Kombination von endlosfaserverstärkten Halbzeugen mit lang- oder kurzfaserverstärkten Materialien. So wird beispielsweise bei der Durchlade ein endlosfaserverstärktes Halbzeug im Pressverfahren vorgeformt und anschließend eine Rippenstruktur aus kurzfaserverstärktem Granulat im Spritzgießprozess angespritzt. Dadurch kann die Steifigkeit des Bauteils deutlich erhöht und zusätzliche Verstärkungs- und Anbindungselemente können integriert werden. Untersuchungen an einer hochsteifen endlosfaserverstärkten Topologiestruktur in Hybridbauweise $(\mathrm{HTH})$ des Instituts für Strukturleichtbau der TU Chemnitz bestätigen den hohen Leichtbaugrad durch diese Hybridbauweise und zeigen eine deutliche Erhöhung der Leichtbaukennzahl, ein Verhältnis des relativen Steifigkeitszuwachses bei 3-Punkt-Biegung zum relativen Massezuwachs, bei Kombination eines unverstärkten Polypropylens und eines endlosfaserverstärkten CFK-Einlegers [13]. Um die Biege- und Torsionssteifigkeit des Schwellerprofils zu erhöhen, wird daher ein solch hybrides Werkstoffkonzept, bestehend aus einem endlosfaserverstärkten, zum Hutprofil umgeformten PA66 CF60 mit angespritzten Verstärkungsrippen aus einem langfaserverstärktem PA66 CF40, entwickelt. Die Rippenstruktur erhöht dabei die Steifigkeit des Profils, hält die Profiloberseite und -unterseite auf Abstand und überträgt, wie auch der Schaumkern des Referenzbauteils, die dazwischen auftretenden Schubkräfte. Die obere Profilseite wird durch das Auftreffen des Impaktors auf Druck, die untere Seite auf Zug beansprucht [14]. Um die auftretenden Zugkräfte an der Unterseite des Profils aufnehmen zu können, wird das Schwellerprofil mit einem endlosfaserverstärkten TP-FKVDeckel, ebenfalls aus PA66 CF60, verschlossen. Aufgrund des Fertigungsprinzips ergibt sich eine zweischalige Bauweise, da der Deckel nachträglich an das Bauteil gefügt werden muss und nicht direkt in einem Prozessschritt angebunden werden kann (siehe $A b b .2$ ). 
Als Fügetechnologie wird, in Anlehnung an das Fügekonzept des BMW i3 und BMW i8, eine Verklebung mit einem Zweikomponenten-Polyurethanstrukturklebstoff [15] zu Grunde gelegt.

\section{Bauweisenkonzept Schwellerprofil:}

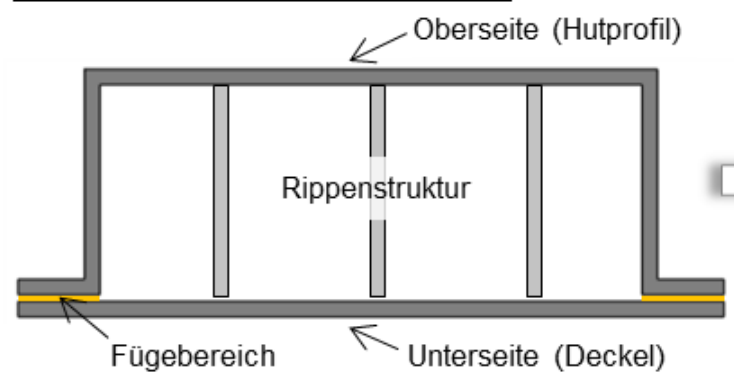

- TP-FKV, Kohlenstofffaser, Langfaser

TP-FKV, Kohlenstofffaser, Endlosfaser

Klebstoff

\section{Fertigungsgerecht gestaltetes Schwellerprofil:}

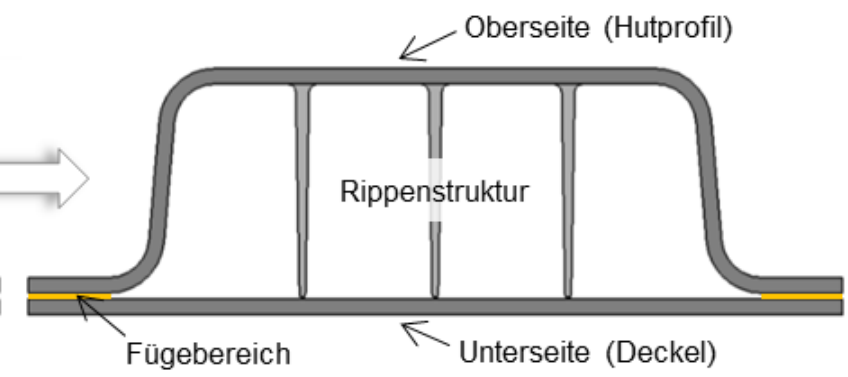

Abbildung 2: Bauweisenkonzept und fertigungsgerecht gestaltetes TP-FKV-Schwellerprofil [12]

Die grundsätzliche Gestaltung des Lagenaufbaus für das Hutprofil und den Deckel orientiert sich in Bezug auf die Faserorientierungen im Laminat am Materialbaukasten der Karosseriestrukturbauteile des BMW i3, BMW i8, BMW 7er (siehe [2]) und an der am Schweller auftretenden Biege- und Torsionsbelastung (siehe Kap. 2). Aufgrund dieser Anforderungen und Rahmenbedingungen wird für die Bauteilauslegung ein Lagenaufbau von $\left[+45^{\circ} / 0^{\circ} /-45^{\circ} / 0^{\circ}\right]_{s}$ zu Grunde gelegt. Der beschriebene Lastfall wird in der FE-Berechnung vereinfacht als quasistatische Belastung angenommen und das Versagen anhand des first ply failure (FPF) bewertet.

Anhand des ideellen Flächenträgheitsmomentes, des beschriebenen Lastfalls und der dafür erforderlichen Kraft- und Wegkriterien ergibt sich eine Mindestwandstärke des Hutprofils von 4,9 mm mit insgesamt 35 Einzellagen und einem Lagenaufbau von [ $+45^{\circ}{ }_{2} / 0^{\circ} 7 /-45^{\circ}{ }_{2} / 0^{\circ}{ }_{13} /-45^{\circ}{ }_{2} / 0^{\circ}{ }_{7} /+45^{\circ}{ }_{2}$ ]. Der Deckel hingegen benötigt nur $3,8 \mathrm{~mm}$ mit insgesamt 28 Einzellagen und einem Lagenaufbau von $\left[+45^{\circ}{ }_{2} / 0^{\circ} 7 /-45^{\circ}{ }_{2} / 0^{\circ} 6 /-45^{\circ}{ }_{2} / 0^{\circ} 7 /+45^{\circ}{ }_{2}\right]$. 


\section{Halbzeugherstellung für experimentelle Untersuchungen}

Für die Herstellung des Hutprofils und des Deckels muss zuerst in vorgelagerten Prozessschritten die Halbzeugherstellung aus vorimprägnierten UD-Tapes erfolgen. Dabei handelt es sich um gespreizte und mit Matrix getränkte Kohlenstofffasern ohne zusätzliche textile Hilfsstoffe, wie Näh- und Wirkfäden. Der schichtweise Aufbau der Einzellagen zu Layups erfolgt mittels Legeverfahren, welche eine freie Wahl der Faserorientierung zulassen und so eine Ausrichtung der Fasern an die jeweiligen funktionalen Anforderungen ermöglichen. Im Allgemeinen wird zwischen zwei verschiedenen Verfahrensvarianten unterschieden. Einerseits können die vorimprägnierten Tapes unmittelbar am Legekopf erwärmt und direkt beim Ablegen über Andrückrollen konsolidiert werden. Diese Direktkonsolidierung vermeidet zwar einen zusätzlichen Konsolidierungsprozess, reduziert aber auch die Ablagegeschwindigkeit der Tapes [16]. Die Ablagerate ist dabei abhängig von der verwendeten Wärmequelle zum An- bzw. Aufschmelzen der Matrix der vorimprägnierten Tapes unmittelbar vor deren Ablage $[17,18]$. Im Vergleich zu anderen Wärmequellen, wie beispielsweise Heißgas und Infrarot, besitzt der Laser als Energiequelle die höchste Energiedichte und die geringste Ansprechzeit zur Erwärmung der Tapes. In Bezug auf die Angaben von $[16,18,19,20]$ ist dabei ein Materialdurchsatz von bis zu $50 \mathrm{~kg} / \mathrm{h}$ möglich. Andererseits besteht die Möglichkeit, wie beispielsweise bei der Technologie RELAY®, die UD-Tapes nur punktuell mittels Ultraschall zueinander zu fixieren, um dadurch die Ablegerate gegenüber der Direktkonsolidierung auf bis zu $350 \mathrm{~kg} / \mathrm{h}$ zu erhöhen [21]. In einem nachgelagerten Prozessschritt ist jedoch die Konsolidierung der UD-Tapes unter Einwirkung von Druck und Temperatur erforderlich. Zur Konsolidierung können sowohl kontinuierliche Prozesse mittels Doppelbandpressen als auch diskontinuierliche Prozesse mit statischen Heizpressen verwendet werden (siehe $A b b$. 3). Um dabei eine möglichst große Menge an Material herstellen zu können, empfiehlt sich für eine Großserienanwendung grundsätzlich ein fortlaufender Prozess.

Anlagenschema: Doppelbandpresse

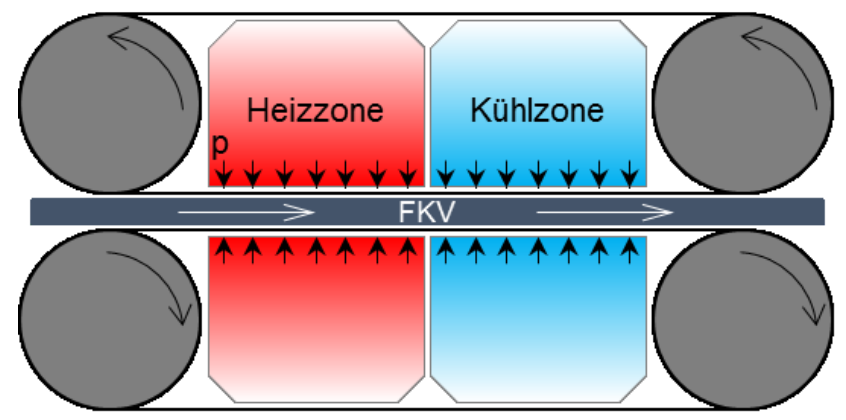

Anlagenschema: statische Heizpresse

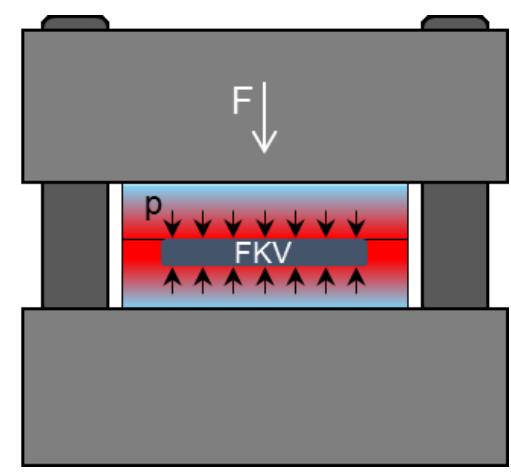

Abbildung 3: Anlagenschema einer isobaren Doppelbandpresse (links) und einer statischen Heizpresse (rechts) zur Konsolidierung der Layups

\subsection{Konsolidierung mittels Doppelbandpresse}

Aufgrund dessen erfolgte die Konsolidierung der in Kap. 3 beschriebenen Lagenaufbauten auf einer isobaren Doppelbandpresse der Fa. Held Technologie $\mathrm{GmbH}$. Es handelt sich dabei um eine Presse mit zwei kontinuierlich rotierenden Endlosstahlbändern mit dazwischenliegenden Druckplatten, welche sich im Vergleich $z u$ anderen Doppelbandpressen für die Verarbeitung von derartigen Hochleistungswerkstoffen mit Prozesstemperaturen von bis zu $400{ }^{\circ} \mathrm{C}$ und 80 bar eignet [22]. Um einen konstanten Dichtungsdruck in der Anlage zu gewährleisten und das Laminat während des Konsolidierungsprozesses zu stabilisieren, wurden Formrahmen aus Aluminium gemeinsam mit den Layups durch den Prozess geführt. Die Hauptfaserorientierung der Layups in $0^{\circ}$ entsprach dabei der Produktionsrichtung der Doppelbandpresse, die Layupabmaße von $805 \mathrm{~mm}$ x 600 mm orientierten sich an einem möglichst geringen Materialverschnitt der Platinen.

Im Rahmen der experimentellen Untersuchungen wurden die Prozessparameter "Druck“ und "Temperatur" jeweils variiert und die Vorschubgeschwindigkeit mit $0,3 \mathrm{~m} / \mathrm{min}$ als konstant angenommen (siehe Tab. 2). Unabhängig von den gewählten Prozessparametern konnte jedoch kein zufriedenstellendes Ergebnis in Bezug auf die Laminatqualität der Platinen erzielt werden. Die Qualität des 
Laminats wurde dabei anhand einer optischen und einer Ultraschalluntersuchung beurteilt. Es zeigte sich bei allen Laminaten ein stark abgeschwächtes Ultraschallsignal, was auf einen hohen Porengehalt und Delaminationen im Laminat hinweist. Aufgrund der Lagenanzahl von bis zu 35 Einzellagen kann, trotz des schrittweisen Einziehens der Layups zwischen die beiden Stahlbänder, die vorhandene Luft nicht vollständig aus dem Laminat entweichen. Obwohl die Hilfsrahmen zur Stabilisierung eingesetzt wurden, zeigten sich zudem Verschiebungen der $0^{\circ}$-Lagen, welche sich in Form von Welligkeiten im Laminat und einem Fließen quer zur $0^{\circ}$-Faserorientierung zeigten. Dies lässt sich durch die hohe Anzahl an $0^{\circ}$-Lagen im Laminat und die dadurch fehlende Querstabilisierung dieser Lagen durch Interaktion mit benachbarten Schichten erklären. Im Vergleich zu den beschriebenen Layups mit einer hohen Vorzugsrichtung in $0^{\circ}$ konnte beispielsweise bei einem orthotropen Layup mit einer abwechselnden Faserorientierung von $0^{\circ} / 90^{\circ}$ und einer Wandstärke von 2,0 mm auf der isobaren Doppelbandpresse der Fa. Held Technologie $\mathrm{GmbH}$ eine äußerst gute Laminatqualität erzielt werden. Dadurch wird die Abhängigkeit des Konsolidierungsergebnisses von der Art des Lagenaufbaus und der zu erreichenden Laminatdicke deutlich und die grundsätzliche Fähigkeit eines kontinuierlichen Fertigungsprozesses mit einer derartigen Doppelbandpresse bestätigt.

Tabelle 2: Übersicht der Layups, der Prozessparameter und der Konsolidierungsergebnisse der experimentellen Untersuchungen auf der Doppelbandpresse

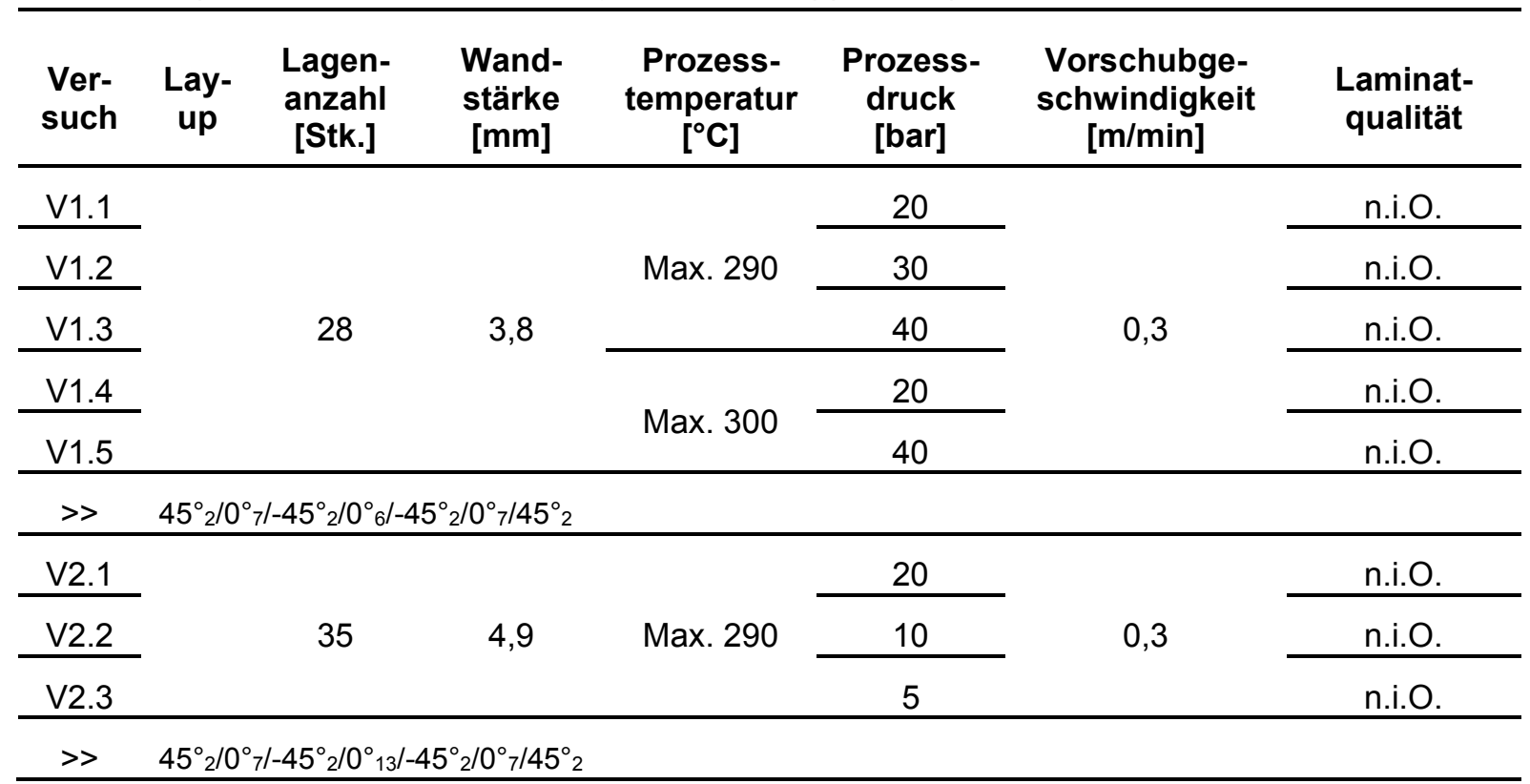




\subsection{Konsolidierung mit statischer Laborplattenpresse}

Um den Einfluss des Fertigungsprinzips auf die Laminatqualität weiter zu untersuchen, wurde zum Vergleich ein Konsolidierungsversuch auf einer statischen Laborplattenpresse von Fa. Dr. Collin GmbH mit einer Probeplattengröße von 260 mm x 260 mm am Institut für Strukturleichtbau der TU Chemnitz durchgeführt. Die Layups wurden dabei in das Unterwerkzeug gelegt und aufgrund der Form der Kavität analog zu den Formrahmen aus den vorher beschriebenen Versuchen auf der Doppelbandpresse durch diese umlaufend begrenzt. Zur Konsolidierung der Layups wurde jeweils das gleiche Druckprofil mit bis zu 15 bar Druck und dasselbe Temperaturprofil mit bis zu $290^{\circ} \mathrm{C}$ und 24 min Zykluszeit verwendet (siehe Tab. 3). Wie auch bei den Versuchen auf der Doppelbandpresse konnte für die Layups (siehe Kap. 3) keine ausreichend gute Laminatqualität erzielt werden. Sowohl ungetrocknete als auch bei $80^{\circ} \mathrm{C}$ und $6 \mathrm{~h}$ vorgetrocknete Layups (siehe V3.1 bis V4.2 in Tab. 3) weisen in der mikroskopischen Analyse von Schliffbildern in $0^{\circ}$-Faserorientierung vertikale Risse (siehe $A b b .4$ ) und bei der Ultraschallprüfung ein stark abgeschwächtes Signal auf. Die Ergebnisse beider Analysemethoden deuten auf Delaminationen und Lufteinschlüsse im Laminat hin.
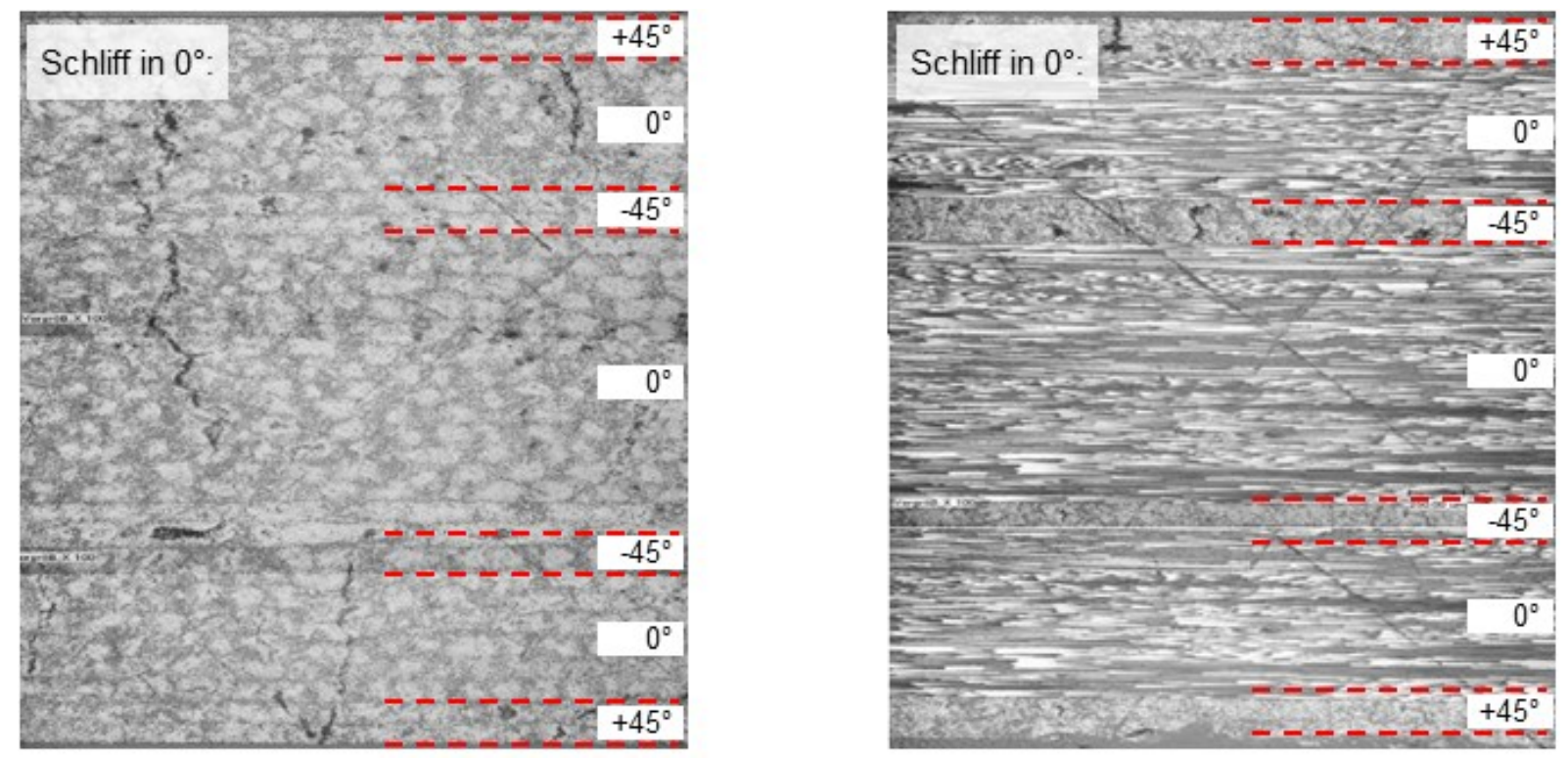

Abbildung 4: Mikroskopiebilder eines ungetrockneten (V4.1, links) und eines vorgetrockneten (V4.2, rechts) PA66 CF60-Laminats

Aufgrund dessen kann weder der Konsolidierungsprozess mit einer statischen Presse noch der mit einer Doppelbandpresse ursächlich für die Qualität der gewählten Layups sein. Wie Tab. 3 zeigt ist jedoch ein Einfluss des Lagenaufbaus auf das Ergebnis der Konsolidierung zu erkennen. 
Tabelle 3: Übersicht der Layups, der Prozessparameter und der Konsolidierungsergebnisse des experimentellen Versuchs auf der Laborplattenpresse

\begin{tabular}{|c|c|c|c|c|c|c|c|}
\hline $\begin{array}{l}\text { Ver- } \\
\text { such }\end{array}$ & $\begin{array}{l}\text { Lay- } \\
\text { up }\end{array}$ & $\begin{array}{l}\text { Lagen- } \\
\text { anzahl } \\
\text { [Stk.] }\end{array}$ & $\begin{array}{l}\text { Wand- } \\
\text { stärke } \\
{[\mathrm{mm}]}\end{array}$ & $\begin{array}{c}\text { Prozess- } \\
\text { temperatur }\left[{ }^{\circ} \mathrm{C}\right]\end{array}$ & $\begin{array}{l}\text { Prozess- } \\
\text { druck } \\
\text { [bar] }\end{array}$ & $\begin{array}{l}\text { Presszeit } \\
\text { [min] }\end{array}$ & $\begin{array}{l}\text { Laminat- } \\
\text { qualität }\end{array}$ \\
\hline V3.1 & & 28 & 3,8 & Max. 290 & 15 & 24 & n.i.O. \\
\hline & \multicolumn{7}{|c|}{$45^{\circ}{ }_{2} / 0^{\circ} 7 /-45^{\circ}{ }_{2} / 0^{\circ}{ }_{6} /-45^{\circ}{ }_{2} / 0^{\circ} 7 / 45^{\circ}{ }_{2}$} \\
\hline V3.2 & & 28 & 3,8 & $\begin{array}{c}\text { Max. } 290 \\
\text { (vorgetrocknet } \\
\text { bei } 80{ }^{\circ} \mathrm{C} \& 6 \mathrm{~h} \text { ) }\end{array}$ & 15 & 24 & n.i.O. \\
\hline & \multicolumn{7}{|c|}{$45^{\circ}{ }_{2} / 0^{\circ} 7 /-45^{\circ} 2 / 0^{\circ} 6 /-45^{\circ}{ }_{2} / 0^{\circ} 7 / 45^{\circ}{ }_{2}$} \\
\hline V4.1 & & 35 & 4,9 & Max. 290 & 15 & 24 & n.i.O. \\
\hline & \multicolumn{7}{|c|}{$45^{\circ}{ }_{2} / 0^{\circ}{ }_{7} /-45^{\circ}{ }_{2} / 0^{\circ}{ }_{13} /-45^{\circ}{ }_{2} / 0^{\circ}{ }_{7} / 45^{\circ}{ }_{2}$} \\
\hline V4.2 & & 35 & 4,9 & $\begin{array}{c}\text { Max. } 290 \\
\text { (vorgetrocknet } \\
\text { bei } 80^{\circ} \mathrm{C} \& 6 \mathrm{~h} \text { ) }\end{array}$ & 15 & 24 & n.i.O. \\
\hline & \multicolumn{7}{|c|}{$45^{\circ}{ }_{2} / 0^{\circ}{ }_{7} /-45^{\circ}{ }_{2} / 0^{\circ}{ }_{13} /-45^{\circ}{ }_{2} / 0^{\circ}{ }_{7} / 45^{\circ}{ }_{2}$} \\
\hline V4.3 & & 35 & 4,9 & Max. 290 & 15 & 24 & n.i.O. \\
\hline & \multicolumn{7}{|c|}{$45^{\circ}{ }_{1} /-45^{\circ}{ }_{1} / 0^{\circ} 7 / 45^{\circ} 1 /-45^{\circ}{ }_{1} / 0^{\circ}{ }_{13} /-45^{\circ}{ }_{1} 1 / 45^{\circ}{ }_{1} / 0^{\circ}{ }^{\circ} 7 /-45^{\circ}{ }_{1} / 45^{\circ}{ }_{1}$} \\
\hline V4.4 & & 35 & 4,9 & Max. 290 & 15 & 24 & $\begin{array}{c}\text { b.i.O. } \\
\text { (Verzug der } \\
\text { Platte) }\end{array}$ \\
\hline & \multicolumn{7}{|c|}{$45^{\circ}{ }_{1} / 0^{\circ}{ }_{4} / 45^{\circ}{ }_{1} / 0^{\circ}{ }_{3} /-45^{\circ}{ }_{1} / 0^{\circ}{ }_{4} /-45^{\circ} 1 / 0^{\circ}{ }_{5} /-45^{\circ}{ }_{1} / 0^{\circ}{ }_{4} /-45^{\circ}{ }_{1} / 0^{\circ}{ }_{3} / 45^{\circ}{ }_{1} / 0^{\circ}{ }_{4} / 45^{\circ}{ }_{1}$} \\
\hline V4.5 & & 35 & 4,9 & Max. 290 & 15 & 24 & b.i.O. bis i.O. \\
\hline
\end{tabular}

Durch Optimierung des Lagenaufbaus nach den allgemeinen Gestaltungsrichtlinien der klassischen Laminattheorie wurde der Lagenaufbau schrittweise verändert. Ein Austausch von $+45^{\circ}$ und $-45^{\circ}$ Lagen (siehe V4.3 in Tab. 3) bewirkt noch keine qualitative Verbesserung des Laminats. Durch eine bessere Verteilung der $0^{\circ}$-Lagen im Laminat (siehe V4.4 in Tab. 3) und zugleich eine Erhöhung der $+/-45^{\circ}$ Anteile (siehe V4.5 in Tab. 3) kann die Laminatqualität erhöht werden. In Anlehnung an die allgemeinen Gestaltungsrichtlinien von Laminaten nach der klassischen Laminattheorie ist bei den Varianten V4.4 und V4.5 die Anzahl an benachbarten Einzellagen mit gleicher Faserorientierung gegenüber den Varianten V4.1 bis V4.3 reduziert. Dies bewirkt eine merkliche Verbesserung der Laminatqualität (siehe $A b b$. 5), verursacht bei V4.4 jedoch einen Verzug der Probeplatte und verändert bei V4.5 deutlich den ursprünglich erforderlichen Lagenaufbau für das Schwellerprofil und die damit verbundenen mechanischen Eigenschaften (siehe Tab. 4). Im Hinblick auf eine großserielle Fertigung der vorimprägnierten Halbzeuge ist jedoch eine Presszeit von 24 min inakzeptabel. 

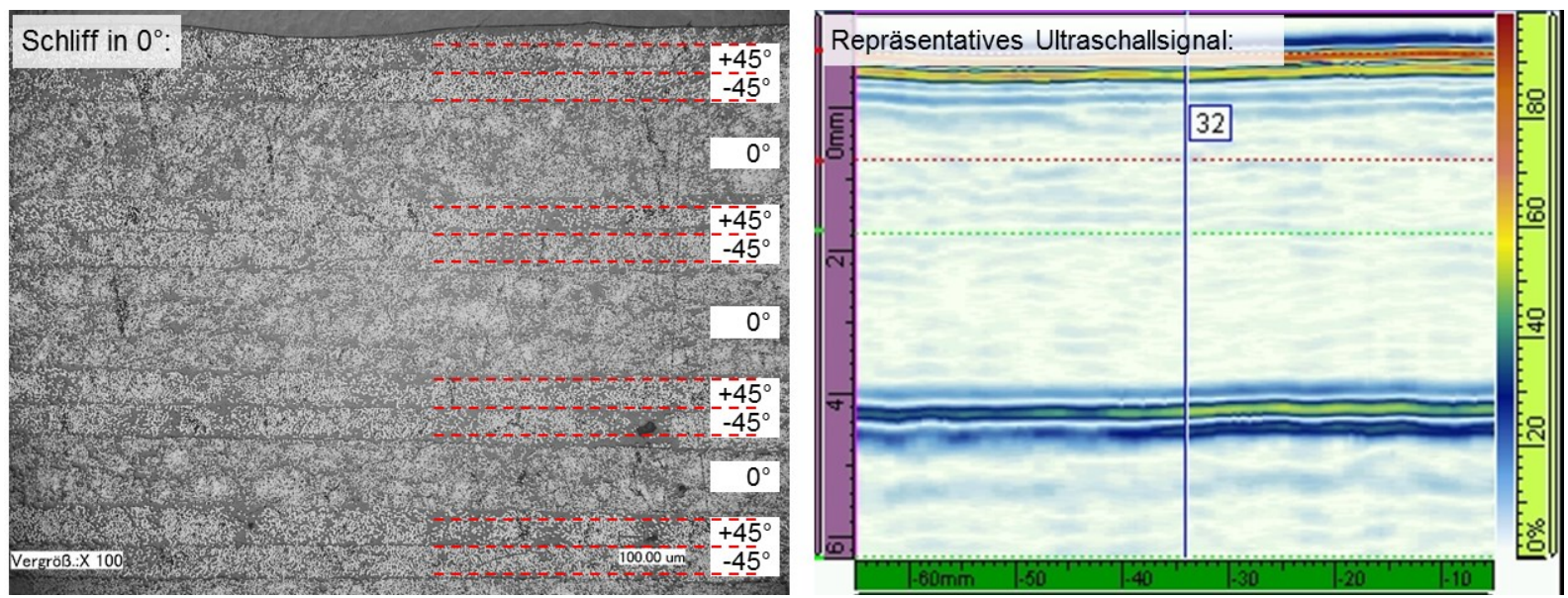

Abbildung 5: Mikroskopiebild in $0^{\circ}$ (links) und repräsentatives Ultraschallsignal in Plattendicke von V4.5

Tabelle 4: Übersicht der mechanischen Eigenschaften E-Modul $E_{1}$, E-Modul $E_{2}$ und Schubmodul $S_{12}$ der Layupvarianten V4.1 bis V4.

\begin{tabular}{cccc}
\hline Versuch & $\mathrm{E}_{\mathbf{1}}[\mathrm{GPa}]$ & $\mathrm{E}_{\mathbf{2}}$ [GPa] & $\mathbf{S}_{\mathbf{1 2}}$ [GPa] \\
\hline $\mathrm{V} 4.1$ & 84,5 & 9,7 & 7,1 \\
\cline { 1 - 2 } V4.2 & 62,0 & 13,5 & 13,0 \\
\hline $\mathrm{V} 4.5$ & & & 9,5 \\
\hline
\end{tabular}

\section{Zusammenfassung und Schlussfolgerung}

Zusammenfassend stellt weder der untersuchte Doppelbandprozess noch der statische Pressprozess hinsichtlich der erzielbaren Laminatqualität und der erforderlichen Anlagenkapazität für das beschriebene Schwellerprofil eine großserientaugliche Fertigungstechnologie dar. Zwar kann grundsätzlich die Konsolidierungszeit auf der statischen Presse noch verkürzt werden, jedoch ist hierbei keine Reduzierung auf unter 10 min Zykluszeit zu erwarten. Der Doppelbandprozess eignet sich aufgrund seines kontinuierlichen Fertigungsprinzips grundlegend besser für eine Produktion in Großserie. Jedoch gilt es, die beschriebenen Mängel durch eine Optimierung der Layupführung durch die Anlage und durch eine Anpassung der Prozessparameter, beispielweise durch Veränderung des Temperaturprofils zwischen Heiz- und Kühlzone, zu beseitigen. Dabei sollte vor allem ein Augenmerk auf die Prozessgeschwindigkeit gelegt werden, da eine Vorschubgeschwindigkeit von $0,3 \mathrm{~m} / \mathrm{min}$ den Durchsatz im Vergleich zu den in Kap. 4 beschriebenen Layuptechnologien mit Direktkonsolidierung zwar um 50 \% erhöht, jedoch für eine Großserienfertigung noch zu gering ist.

\section{Danksagung}

Diese Arbeit entstand durch die finanzielle Unterstützung der BMW Group, durch Unterstützung des Bundesexzellenzclusters EXC 1075 „Technologiefusion für multifunktionale Leichtbaustrukturen“ und wurde teilweise von der Deutschen Forschungsgemeinschaft gefördert. Die Autoren danken für die finanzielle Unterstützung. 


\section{Literaturverzeichnis}

[1] Ahlers, M., Sammer, K.: New BMW 7 series. Carbon Core. EuroCarBody 2015. Bad Nauheim, 21.10.2015.

[2] Hofbauer, D.: Textiltechnik für die automobile Großserienfertigung von Karosseriestrukturbauteilen. 15. Chemnitzer Textiltechnik-Tagung. Chemnitz, 31.05.-01.06.2016.

[3] Obermann, C.: Hybridbauteile mit Organoblechen: Leichtbaupotenzial und Verarbeitungstechnik. In: Drummer, D. (Hrsg.): Thermoplastische Faserverbundkunststoffe, München: Hanser, 2011, S. $51-64$.

[4] Täger, O., Ehleben, M., Lohmann, J., Kharchi, K., Schweizer, K., Kroll, L., Meyer, M., Nendel, W.: New Fiber-Reinforced Thermoplastic Metal Hybrids - a New Technology Concept for a Highly Crash Loaded Lightweight Electric Vehicle Component. In: Borgmann, H. (Hrsg.): ITHEC 2014 Manuscript A6, 2014, S. 32-33.

[5] Philipp, S.: Die Kunst der Verstärkung, BASF SE. Ludwigshafen, 21. 03. 2012.

[6] Steinebach, M.: Leichtbau schützt die Umwelt und erhöht die Sicherheit im Auto, Technische Universität Chemnitz; Bundesexzellenzcluster MERGE. Chemnitz, 28.03.2017.

[7] Hack, C.: Leicht und serienreif. In: Automobilindustrie Insight, Sonderausgabe 07/2016, S. 32-34

[8] Gonter, M., Schwarz, T., Seiffert, U., Zobel, R.: Fahrzeugsicherheit - dynamische Fahrzeugkollision. In: Braess, H.-H., Seiffert, U. (Hrsg.): Vieweg Handbuch Kraftfahrzeugtechnik, Springer Vieweg, 2013, S. 1004-1009.

[9] Teske, L., Goßmann, H.: Karosseriebauweisen. In: Braess, H.-H., Seiffert, U. (Hrsg.): Vieweg Handbuch Kraftfahrzeugtechnik, 2013, Springer Vieweg, S. 498-540.

[10] Dirschmid, F., Weiss, T.: Die CFK-Karosserie des BMW i8 und deren Auslegung. In: Tecklenburg, G. (Hrsg.): Karosseriebautage Hamburg, 2014, S. 217-231.

[11] Hofbauer, D., Miadowitz, T., Dix, M., Gustke, K., Kroll, L., Kaufmann, J.: Auslegung und Simulation von Faser-Kunststoff-Verbundprofilen mit hybriden Textil- und Kernstrukturen. In: Huber, O., et al. (Hrsg.): 7. Landshuter Leichtbau-Colloquium (2015), 2015, S. 184-196.

[12] Hofbauer, D.: Bauweisenkonzept TP-FKV. BMW Group, Landshut, 22.10.2014.

[13] Kroll, L., Nossol, P., Tröltzsch, ., Wu, W., Ziegmann, G.: Fertigung von Technologiedemonstratoren. In: Cherif, C. (Hrsg.): Leichtbau mit Textilverstärkung für Serienanwendungen, Auerbach: Wissenschaftliche Scripten, 2013, S. 339-377.

[14] Hofbauer, D., Gustke, K., Kaufmann, J., Götze, U., Schmidt, A.: Scoring concept for fibre reinforced composites used in mass production car bodies. In: Kroll, L., et al. (ed.): 2nd International MERGE Technologies Conference, Auerbach: Wissenschaftliche Scripten, 2015, pp. 165-174.

[15] Lutz, A., Schmatloch, S.: Composites strukturell kleben. adhäsion KLEBEN \& DICHTEN 58(9) (2014), S. 32-37.

[16] Champa, D.: Automated Composites Manufacturing Review. Manufacturing with composites 2012. Charleston, SC, USA, 23.-24.10.2012.

[17] Haake, J. M.: High power diode laser-assisted fiber placement of composite structure. Westec Conference. Los Angeles, Kalifornien, USA, 04.-07.04.2005.

[18] Schledjewski, R., Schlottermüller, M., Neitzel, M., Beresheim, G., Mack, J., Brzeski, M.: Wickelund Legetechnik. In: Neitzel, M., et al. (Hrsg.): Handbuch Verbundwerkstoffe, 2014, S. 325-356.

[19] Stokes-Griffin, C. M., Compston, P.: An inverse model for optimisation of laser heat flux distributions in an automated laser tape placement process for carbon-fibre/PEEK. In: Composites Part A: Applied Science and Manufacturing 88 (2016), pp. 190-197.

[20] Szcesny, M., Heieck, F., Carosella, S., Middendorf, P., Sehrschön, H., Schneiderbauer, M.: The advanced ply placement process - an innovative direct 3D placement technology for plies and tapes. Advanced Manufacturing: Polymer \& Composites Science 3(1) (2017), pp. 2-9. doi:10.1080/20550340.2017.1291398

[21] Dieffenbacher GmbH: Fiberforge 4.0 - The world`s fastest tape laying system. Eppingen, Oktober 2016.

[22] Held Technologie GmbH: Doppelbandpressen von HELD. Hocheffiziente Produktion in TopQualität. Trossingen, 2017. 